\title{
THE GROUP OF AUTOMORPHISMS OF THE MODULI SPACE OF PRINCIPAL BUNDLES WITH STRUCTURE GROUP $F_{4}$ AND $E_{6}$
}

\author{
ÁLVARO ANTÓN SANCHO
}

\begin{abstract}
Let $X$ be a smooth complex projective irreducible curve of genus $g \geq 3$. Let $G$ be the simple complex exceptional Lie group $F_{4}$ or $E_{6}$ and let $M(G)$ be the moduli space of principal $G$-bundles. In this work we describe the group of automorphisms of $M(G)$. In particular, we prove that the only automorphisms of $M\left(F_{4}\right)$ are those induced by the automorphisms of the base curve $X$ by pull-back and that the automorphisms of $M\left(E_{6}\right)$ are combinations of the action of the automorphisms of $X$ by pull-back, the action of the only nontrivial outer involution of $E_{6}$ on $M\left(E_{6}\right)$ by taking the dual and the action of the third torsion of the Picard group of $X$ by tensor product. We also prove a Torelli type theorem for the moduli spaces of principal $F_{4}$ and $E_{6}$-bundles, which we use as an auxiliary result in the proof of the main theorems, but which is interesting in itself. We finally draw some conclusions about the way we can see the natural map $M\left(F_{4}\right) \rightarrow M\left(E_{6}\right)$ induced by the inclusion of groups $F_{4} \hookrightarrow E_{6}$.
\end{abstract}

\section{INTRODUCTION}

Let $X$ be a smooth complex projective irreducible curve of genus $g \geq 3$ and let $G$ be a complex reductive Lie group. Principal $G$-bundles over $X$ are geometric objects which come with an action of the group of transformations or structure group, $G$. The set of isomorphism classes of principal $G$-bundles is parametrized by the cohomology set $H^{1}(X, G)$. Using Mumford's GIT [16], Ramanathan [18, 19, 20] gave a notion of stability for principal $G$-bundles in order to construct the moduli space of polystable principal $G$-bundles, $M(G)$, as a coarse moduli scheme whose points correspond bijectively to isomorphism classes of polystable $G$-bundles. This construction works when $g \geq 2$, but we will consider $g \geq 3$ for technical reasons in the proof of the main theorem of Section 5 .

These spaces have a very rich topology and geometry and have been intensively studied in mathematics and theoretical physics. A way to study the moduli space $M(G)$ is to describe the subvarieties and automorphisms of $M(G)$, in

2010 Mathematics Subject Classification. Primary 14H60; Secondary 57R57.

Key words and phrases. Principal bundle, Moduli space, Exceptional group, Automorphism, Torelli theorem. 
the spirit of Serman 22 and Kouvidakis and Pantev [15]. Serman [22], for example, proves that the forgetful morphisms $M(\mathrm{O}(n, \mathbb{C})) \rightarrow M(\mathrm{GL}(n, \mathbb{C}))$ and $M(\mathrm{Sp}(2 n, \mathbb{C})) \rightarrow M(\mathrm{SL}(2 n, \mathbb{C}))$ are closed immersions, so they define subvarieties which are isomorphic to $M(\mathrm{O}(n, \mathbb{C}))$ and $M(\operatorname{Sp}(2 n, \mathbb{C}))$ respectively. It is also interesting to study the group of automorphisms of this kind of moduli spaces. This problem connects with the work of Kouvidakis and Pantev [15]. They determined the group of automorphisms of the moduli space in the particular case of vector bundles with a given rank and degree. They proved that any automorphism of $M(\mathrm{SL}(n, \mathbb{C}))$ is a combination of the automorphisms of the moduli space which comes from the outer involution of the Dynkin diagram, $E \mapsto E^{*}$, with the automorphisms which consists in tensoring by a line bundle of order $n$ and the automorphisms which come from automorphisms of the base curve, $X$. In [14] the same result is proved with other techniques that involve Hecke curves, which are minimal rational curves coming from Hecke transformations. Other authors have recently worked along this path dealing with other particular groups (for example, Biswas, Gómez and Muñoz [5, 6]). Using the perspective of [14], Biswas, Gómez and Muñoz [6] give a complete description of the group of automorphisms of $M(\operatorname{Sp}(2 n, \mathbb{C}))$. They prove that these automorphisms combine automorphisms of the base curve with those induced by line bundles of order two. Observe that in this case there are no automorphisms of the moduli space coming from outer automorphisms of the group because the Dynkin diagram has no symmetries.

In this work, we study the case in which the structure group $G$ is $F_{4}$ or $E_{6}$, both simple complex Lie groups of exceptional type. We focus on these groups because our arguments use strongly the form of the homogeneous invariant polynomials of the groups. These arguments are not generalizable to other groups (for example, to orthogonal groups). For the rest of the groups of type $E$, it would also be needed a specific description of the parabolic subgroups of the group, which we have not found in the literature, in order to give a concrete notion of stability for the corresponding principal bundles. In particular, we prove that the automorphisms of $M\left(F_{4}\right)$ are exactly those induced by the automorphisms of the curve $X$ and that every automorphism of $M\left(E_{6}\right)$ is a combination of an automorphism of $X$, the action on $M\left(E_{6}\right)$ of an outer automorphism of $E_{6}$ and the tensor product by an element of the third torsion of the Picard group of $X$ (the last automorphisms are induced by the action of $H^{1}\left(X, Z\left(E_{6}\right)\right)$ on $M\left(E_{6}\right)$, where $Z\left(E_{6}\right) \cong \mathbb{Z}_{3}$ is the center of $\left.E_{6}\right)$. Observe that these two results are analogous, since $F_{4}$ is centerless and it admits no nontrivial outer automorphisms. The conjecture is that for any complex reductive Lie group $G$, every automorphism of the moduli space of principal $G$ bundles, $M(G)$, is a combination of the action on $M(G)$ of an outer automorphism of $G$, the action of an element of $H^{1}(X, Z(G))$, where $Z(G)$ denotes the center of $G$, and the action of an automorphism of the base curve by pull-back. This conjecture has only been proved, as far as we know, for the special linear and general linear groups in [15] and for symplectic groups in [5]. 
In Theorem 3 we prove that the group of automorphisms of $M\left(F_{4}\right)$ is exactly $\operatorname{Aut}(X)$, which acts on $M\left(F_{4}\right)$ by taking the pull-back of the bundles. In Theorems 4 and 5 we reason similarly to how we did with the case of $F_{4}$ and we prove that the group of automorphisms of $M\left(E_{6}\right)$ is isomorphic to $\operatorname{Pic}(X)[3] \rtimes\left(\operatorname{Out}\left(E_{6}\right) \times\right.$ $\operatorname{Aut}(X))$, where $\operatorname{Pic}(X)[3]$ denotes the third torsion of the Picard group of $X$ and it acts on $M\left(E_{6}\right)$ by tensor product, $\operatorname{Out}\left(E_{6}\right) \cong \mathbb{Z}_{2}$ is the group of outer automorphisms of $E_{6}$ and the only notrivial outer involution in $\operatorname{Out}\left(E_{6}\right)$ acts on $M\left(E_{6}\right)$ by taking the dual bundle. The group $\operatorname{Aut}(X)$ also acts on $M\left(E_{6}\right)$ by taking the pull-back. Finally, this description of the groups of automorphisms will help us to obtain some consequences about how we can see $M\left(F_{4}\right)$ included in $M\left(E_{6}\right)$ (Propositions 8 and 9 ). To do all this we first prove a Torelli theorem for $M\left(F_{4}\right)$ and $M\left(E_{6}\right)$ (Theorems 1 and 2 , which says that the moduli space determines the base curve, in the sense that if $X$ and $X^{\prime}$ are complex algebraic curves of genus $g$ and $g^{\prime}$, respectively, with $g, g^{\prime} \geq 3, G$ is $F_{4}$ or $E_{6}, M(G)$ is the moduli space of principal $G$-bundles over $X$ and $M^{\prime}(G)$ is the moduli space of principal $G$-bundles over $X^{\prime}$, then if $M(G) \cong M^{\prime}(G)$, the corresponding base curves are also isomorphic, $X \cong X^{\prime}$. This result, which is interesting in itself, is also relevant to prove Theorems 3 and 4

The paper is organized as follows. Section 2 gives a description of the groups $F_{4}$ and $E_{6}$, emphasizing some facts like the algebra of invariant homogeneous polynomials and a concrete description of the filtrations induced on the bundles by maximal parabolic subgroups of the groups. These facts are relevant in order to present, in Section 3 , an appropriate notion of stability of principal $F_{4}$ and $E_{6^{-}}$ bundles. Section 4 is devoted to describe the Hitchin integrable system associated to $M\left(F_{4}\right)$ and $M\left(E_{6}\right)$. We also define an action of $\mathbb{C}^{*}$ on that system and prove some auxiliary results which will be useful in the proofs of the main results of the paper. In Section 5 we prove the Torelli theorem for $M\left(F_{4}\right)$ and $M\left(E_{6}\right)$ (Theorems 1 and 2 and in Sections 6 and 7 , we give the precise description of the group of automorphisms of $M\left(F_{4}\right)$ (Theorem 3$)$ and $M\left(E_{6}\right)$ (Theorem 5), respectively. Finally, Section 8 is devoted to study some consequences of our description of the groups of automorphisms on the geometry of $M\left(E_{6}\right)$.

\section{The GRoups $F_{4}$ AND $E_{6}$}

We are interested in the group $E_{6}$, the only exceptional simple complex Lie group which admits nontrivial outer automorphisms, and in $F_{4}$, another exceptional complex Lie group which can be seen in a natural way as a subgroup of $E_{6}$. In [1] and 24, a good review about exceptional groups can be read. Ferrar [11, 12 ] also provides a good framework about the group $E_{6}$. Also in [3] some of the topics treated here for the group $E_{6}$ are described.

We consider any complex algebra, $\mathcal{A}$. We will suppose that $\mathcal{A}$ is non-commutative. A classical example is the algebra of $k \times k$ matrices over the complex numbers, with $k \geq 2$, equipped with the natural product of matrices, which is an associative and non-commutative complex algebra. In the algebra $\mathcal{A}$, the Jordan product $\circ$ is 
defined to be the anti-commutator of the product of the algebra $\mathcal{A}$, that is,

$$
A \circ B=\frac{1}{2}(A B+B A)
$$

for $A, B \in \mathcal{A}$. This Jordan product induces in $\mathcal{A}$ the structure of a (probably non-associative) algebra which satisfies the Jordan identity, that is,

$$
(A \circ B) \circ(A \circ A)=A \circ(B \circ(A \circ A))
$$

for any elements $A$ and $B$ of the algebra $\mathcal{A}$. This algebra is called Jordan algebra.

Jordan algebras have been intensively studied and completely classified. In particular, a Jordan algebra which is constructed by introducing the $\circ$ product to an associative algebra $\mathcal{A}$ is called special Jordan algebra; if the original algebra $\mathcal{A}$ is non-associative, it is called exceptional Jordan algebra. It can be proved that there is only one simple exceptional Jordan algebra and that this exceptional algebra is finite-dimensional. It is called Albert algebra ([23, Sec. 5.8]) and may be constructed by introducing the Jordan product in the algebra $\mathfrak{h}_{3}(\mathbb{O})$ of $3 \times 3$ Hermitian matrices over the algebra of the octonions. The Albert algebra thus defined has dimension 27 .

Consider, from now on, the Albert algebra. The Jordan product gives rise to three maps on this algebra: a linear map a defined by the trace, a bilinear map $b$ defined by $b(A, B)=\operatorname{Tr}(A \circ B)$, and a trilinear map $c$ defined by $c(A, B, C)=$ $\operatorname{Tr}((A \circ B) \circ C)$, where $\operatorname{Tr}$ denotes the trace map. The map $b$ is symmetric, since the Jordan product is commutative. It then induces a quadratic form $q$. From the Jordan identity it can be seen that $c(A, B, C)=c(B, C, A)$. Then the trilinear map $t$ is also symmetric. It then gives rise to the so called norm $n$.

Here, and throughout the text, we will not use the word form to denote antisymmetric maps, since all the multilinear maps that will appear will be symmetric. The word form will then be used to denote symmetric multilinear maps. With this notation, the trilinear form $t$ will be denoted, for convenience to our interests, by $\Omega$.

We may define $E_{6}$ to be the group of linear automorphisms of the complexification of the Albert algebra which preserves the norm $n$. That is,

$$
E_{6}=\left\{\alpha \in \operatorname{GL}\left(\mathfrak{h}_{3}(\mathbb{O})^{\mathbb{C}}\right): n(\alpha(A))=n(A) \forall A \in \mathfrak{h}_{3}(\mathbb{O})\right\}
$$

The norm $n$ gives the determinant of a general element of $\mathfrak{h}_{3}(\mathbb{O})^{\mathbb{C}}$. Therefore, the group $E_{6}$ is exactly the group of determinant preserving linear transformations of $\mathfrak{h}_{3}(\mathbb{O})^{\mathbb{C}}$. Its Lie algebra $\mathfrak{e}_{6}$ is then the Lie algebra given by linear endomorphisms preserving the 3 -form $\Omega$. There are two fundamental well-known characteristics of $E_{6}$ which will be relevant to us:

(1) The center of $E_{6}, Z\left(E_{6}\right)$, is isomorphic to the cyclic group of order three, $\mathbb{Z}_{3}$.

(2) The group $E_{6}$ is simply connected.

The group $E_{6}$ thus defined is the simply connected complex Lie group with Lie algebra $\mathfrak{e}_{6}$. 
We can also define the Lie group $F_{4}$ as the group of automorphisms of the Albert algebra. These automorphisms are precisely those linear maps which preserve the three forms, $a, q$ and $n$. These forms are exactly those which define the minimal polynomial of a general element of $\mathfrak{h}_{3}(\mathbb{O})^{\mathbb{C}}$, so $F_{4}$ contains all linear automorphisms of $\mathfrak{h}_{3}(\mathbb{O})^{\mathbb{C}}$ which preserve the minimal polynomial. It is then clear that $F_{4}$ is a subgroup of $E_{6}$. The complex Lie group $F_{4}$ is then a centerless and simply connected complex Lie group whose Lie algebra, $\mathfrak{f}_{4}$, is then the algebra of derivations of the Albert algebra.

The group $E_{6}$ admits six fundamental representations, whose dimensions are 27, 351, 2925, 351, 27 and 78. The Albert algebra gives rise to a 27-dimensional complex representation of $E_{6}, E_{6} \rightarrow \mathrm{SL}\left(\mathfrak{h}_{3}(\mathbb{O})^{\mathbb{C}}\right)$. This representation, which is a fundamental representation, is irreducible and faithful and it is inequivalent to its dual, which of course is also 27-dimensional irreducible and faithful. In fact, these are the smallest irreducible representations of $E_{6}$. The fundamental 27-dimensional representation of $E_{6}$ restricts to a 26-dimensional representation of $F_{4}$, which is irreducible and in fact the fundamental representation of $F_{4}$. The natural inclusion of groups $\mathrm{SL}(26, \mathbb{C}) \hookrightarrow \mathrm{SL}(27, \mathbb{C})$ is compatible with the inclusion $F_{4} \hookrightarrow E_{6}$.

Since $E_{6}$ is simply connected, there exists only one nontrivial outer automorphism of $E_{6}, \sigma$, which corresponds to the unique nontrivial symmetry of the Dynkin diagram of $\mathfrak{e}_{6}$, of order 2, so $\sigma$ is an involution. Since the Dynkin diagram of the group $F_{4}$ admits no symmetries, the group of outer automorphisms of $F_{4}$ is trivial.

A description of parabolic subgroups of $E_{6}$ and the induced filtrations can be read in [21], following [17]. We briefly recall it here in order to make sense of the notion of stability of principal $E_{6}$-bundles. The group $E_{6}$ is the group of automorphisms of a 27-dimensional complex vector space $V$ equipped with a linear 3 -form, $\Omega$. Any filtration of $V$ induced by a maximal parabolic subgroup of $E_{6}$ is of the form

$$
0 \subseteq V_{0} \subseteq \cdots \subseteq V_{r} \subseteq V
$$

where $V_{0}, V_{1}, \ldots, V_{r}$ are isotropic subspaces of $V$ for the 3 -form $\Omega$. Recall that an isotropic subspace of $V$ is a subspace $W$ such that $\Omega(W, W, W)=0$. It is said to be maximal isotropic if it is not properly contained in other isotropic subspace.

The group $F_{4}$ is the subgroup of $E_{6}$ which consists of automorphisms of $(V, \Omega)$ equipped also with a certain symmetric 2 -form, $\omega$. The group $F_{4}$ is, then, the group of automorphisms of $(V, \Omega, \omega)$. There exists a complex 26-dimensional vector space $W$ such that $V$ projects over $W$ and the 2 -form $\omega$ is nondegenerate on $W$ (this $W$ is given by the fundamental representation of $F_{4}$ and it also inherits the 3 -form $\Omega$ ). The group $F_{4}$ is then a subgroup of $\operatorname{SO}(26, \mathbb{C})$. It is then clear that any filtration of $W$ induced by a proper parabolic subgroup of $F_{4}$ is of the form

$$
0 \subsetneq W_{0} \subsetneq \cdots \subsetneq W_{r} \subseteq W_{r}^{\perp} \subsetneq \cdots \subsetneq W_{0}^{\perp} \subsetneq W
$$

where each $W_{i}$ is an isotropic subspace of $W$ for the bilinear form $\omega$ and the orthogonality $\perp$ is also given by $\omega$. Since this filtration should also be a filtration induced by a parabolic subgroup of $E_{6}$, a maximal parabolic subgroup of $F_{4}$ induces 
a filtration of the form

$$
0 \subsetneq W_{0} \subsetneq W_{1} \subsetneq \cdots \subsetneq W_{r} \subseteq W_{r}^{\perp} \subsetneq \cdots \subsetneq W_{1}^{\perp} \subsetneq W_{0}^{\perp} \subsetneq W
$$

where $W_{0}, W_{1}, \ldots, W_{r}$ are isotropic for $\omega$ and $\Omega$.

Finally, we will study the invariant polynomials of the groups $F_{4}$ and $E_{6}$ for the adjoint action of the group on their Lie algebra. Recall the for any complex semisimple Lie group $G$ with Lie algebra $\mathfrak{g}$, an invariant homogeneous polynomial of degree $d$ for the adjoint representation of $G$ in $\mathfrak{g}, \operatorname{Ad}: G \rightarrow \operatorname{GL}(\mathfrak{g})$, is an element $p \in \operatorname{Sym}^{d} \mathfrak{g}^{*}$ such that

$$
p\left(v_{1}, \ldots, v_{d}\right)=p\left(\operatorname{Ad}(g)\left(v_{1}\right), \ldots, \operatorname{Ad}(g)\left(v_{d}\right)\right)
$$

for all $v_{1}, \ldots, v_{d} \in \mathfrak{g}$ and all $g \in G$. Chevalley [7] proved that the algebra of invariant homogeneous polynomials of $G$ is generated by $r=\operatorname{rk} G$ elements.

Let $G=E_{6}$. A family of generators of the algebra of invariant homogeneous polynomials of $E_{6}$ is given by six polynomials $\left\{p_{2}, p_{5}, p_{6}, p_{8}, p_{9}, p_{12}\right\}$, where the degree of $p_{i}$ is $i$. These polynomials are defined by the following expression: if $A \in \mathfrak{e}_{6}$,

$$
p_{i}(A)=\operatorname{Tr}\left(\wedge^{i} A\right),
$$

where $\operatorname{Tr}$ denotes the trace map and $A$ is seen as an element of $\operatorname{SL}(27, \mathbb{C})$ via the fundamental 27-dimensional representation of $E_{6}$. For any element $A \in \mathfrak{e}_{6}$, the characteristic polynomial of $A$ is

$$
\operatorname{det}(y I-A)=y^{27}+\sum_{r=2,5,6,8,9,12} p_{r}(A) y^{27-r} .
$$

The four polynomials of even degree restrict to invariant homogeneous polynomials of $F_{4}$ and the polynomials $p_{5}$ and $p_{9}$ vanish when restricted to $F_{4}$, so, keeping the same notation, $\left\{p_{2}, p_{4}, p_{6}, p_{12}\right\}$ is a basis of the algebra of invariant homogeneous polynomials of $F_{4}$. We also have that, if $A \in \mathfrak{f}_{4}$, the characteristic polynomial of $A$ is

$$
\operatorname{det}(y I-A)=y^{26}+\sum_{r=2,6,8,12} p_{r}(A) y^{26-r} .
$$

\section{The Moduli SPACE of PRINCIPAL BUndles With STRUCTURE GRoup $F_{4}$ AND $E_{4}$}

Let $X$ be a smooth complex projective irreducible curve of genus $g \geq 3$. A principal $E_{6}$-bundle over $X$ is a complex vector bundle of rank 27 and trivial determinant equipped with a global holomorphic symmetric non-degenerate 3 -form, $\Omega$. Given a principal $E_{6}$-bundle $E$, a subbundle $F$ of $E$ is said to be isotropic if $\Omega(F, F, F)=0$. The isotropic subbundle $F$ is said to be maximal isotropic if it is not properly contained in other isotropic subbundle.

Let $E$ be a principal $E_{6}$-bundle. A reduction of structure group of $E$ to a maximal parabolic subgroup of $E_{6}$ can be seen as a filtration of the form

$$
0 \subsetneq E_{0} \subsetneq E_{1} \subsetneq \cdots \subsetneq E_{r} \subsetneq E
$$


where $E_{0}, \ldots, E_{r}$ are isotropic subbundles of $E$ for $\Omega$, according to (1). A reduction of $E$ to a Levi subgroup of a maximal parabolic subgroup gives rise to a decomposition of $E$ into a direct sum of vector subbundles of the form

$$
E=E_{0} \oplus E_{1} / E_{0} \oplus \cdots \oplus E / E_{r},
$$

where $E_{0}, \ldots, E_{r}$ are isotropic subbundles of $E$ for $\Omega$.

From this, an appropriate notion of stability and polystability for principal $E_{6}$ bundles can be given.

Definition 1. Let $E$ be a principal $E_{6}$-bundle with associated symmetric holomorphic 3-form $\Omega$. Then $E$ is semistable if for every proper isotropic subbundle $E^{\prime}$ of $E$ we have that $\operatorname{deg} E^{\prime} \leq 0$. It is stable if for every proper isotropic subbundle $E^{\prime}$ of $E$ we have that $\operatorname{deg} E^{\prime}<0$. It is polystable if it can be written as a direct sum of vector subbundles

$$
E=E_{0} \oplus E_{1} / E_{0} \oplus \cdots \oplus E / E_{r},
$$

where $E_{0}, \ldots, E_{r}$ are degree 0 isotropic subbundles of $E$ for the form $\Omega$.

The moduli space of polystable principal $E_{6}$-bundles is an algebraic variety of dimension $78(g-1)$ which parametrizes isomorphism classes of polystable principal $E_{6}$-bundles.

Similarly, a principal $F_{4}$-bundle can be seen as a holomorphic vector bundle $E$ of rank 26 equipped with a non-degenerate symmetric holomorphic 3 -form and a non-degenerate symmetric holomorphic bilinear form $\omega$. From 2 it follows that a reduction of structure group of $E$ to a maximal parabolic subgroup of $F_{4}$ gives a filtration of the form

$$
0 \subsetneq E_{0} \subsetneq \cdots \subsetneq E_{r} \subseteq E_{r}^{\perp} \subsetneq \cdots \subsetneq E_{0}^{\perp} \subsetneq E,
$$

where $E_{0}, \ldots, E_{r}$ are proper subbundles of $E$ which are isotropic for $\Omega$ and $\omega$ for all $k=0, \ldots, r$. We then have the following notions of stability and polystability for principal $F_{4}$-bundles.

Definition 2. Let $E$ be a principal $F_{4}$-bundle with associated symmetric holomorphic 3 -form $\Omega$ and symmetric holomorphic 2 -form $\omega$. Then $E$ is semistable if for every proper subbundle $E^{\prime}$ of $E$ which is isotropic for $\Omega$ and $\omega$, we have that $\operatorname{deg} E^{\prime} \leq 0$. It is stable if for every such subbundle $E^{\prime}$ of $E$ we have that $\operatorname{deg} E^{\prime}<0$. It is polystable if it can be written as an orthogonal direct sum of vector subbundles

$$
E=W \oplus\left(E^{\prime} \oplus E^{\prime *}\right),
$$

where $E^{\prime}$ is proper isotropic for $\Omega$ and $\omega, \operatorname{deg} E^{\prime}=\operatorname{deg} W=0$.

The moduli space of polystable principal $F_{4}$-bundles is then an algebraic variety of dimension $52(g-1)$ which parametrizes isomorphism classes of polystable principal $F_{4}$-bundles.

We have a natural map between algebraic varieties $M\left(F_{4}\right) \rightarrow M\left(E_{6}\right)$ given by

$$
E \mapsto E \oplus \mathcal{O},
$$


where $\mathcal{O}$ is the trivial line bundle over $X$. Observe form Definitions 1 and 2 that any strictly polystable principal $F_{4}$-bundle is also strictly polystable when seen as $E_{6}$-bundle via the map (3).

\section{The Hitchin Integrable System of $M\left(F_{4}\right)$ And $M\left(E_{6}\right)$}

Let $X$ be a smooth complex projective irreducible curve of genus $g \geq 3$. Let $M(G)$ be the moduli space of polystable principal $G$-bundles over $X$, where $G$ is a semisimple complex Lie group, and let $M_{*}(G)$ be the moduli space of stable and simple principal $G$-bundles, which is a dense open subset of $M(G)$. The tangent space to $M_{*}(G)$ at a stable and simple principal $G$-bundle $E$ is isomorphic to $H^{1}(X, E(\mathfrak{g}))(\mathfrak{g}$ is the Lie algebra of $G)$, which, by Serre duality, coincides with $H^{0}(X, E(\mathfrak{g}) \otimes K)^{*}$, where $K$ is the cotangent bundle over the curve $X$ and $E(\mathfrak{g})$ denotes the adjoint bundle of $E$. Then the cotangent space of $M_{*}(G)$ at the point corresponding to the bundle $E$ is naturally identified with

$$
T_{E}^{*} M_{*}(G) \cong H^{0}(X, E(\mathfrak{g}) \otimes K) .
$$

The elements of $H^{0}(X, E(\mathfrak{g}) \otimes K)$ are called Higgs fields of the bundle $E$.

The adjoint vector bundle $E(\mathfrak{g})$ is a bundle of Lie algebras which are isomorphic to the Lie algebra $\mathfrak{g}$. Let $p$ be an invariant homogeneous polynomial of degree $d$. Then, it is clear (see [13]) that $p$ defines a map, which we will call also $p$, by acting on the fibers of $E(\mathfrak{g})$ :

$$
p: H^{0}(X, E(\mathfrak{g}) \otimes K) \rightarrow H^{0}\left(X, K^{d}\right) .
$$

Let $p_{d_{1}}, \ldots, p_{d_{k}}$ be a family of generators of the ring of invariant homogeneous polynomials of $\mathfrak{g}(k=\mathrm{rk} \mathfrak{g})$. Then we obtain a map

$$
\mathcal{H}=\left(p_{d_{1}}, \ldots, p_{d_{k}}\right): H^{0}(X, E(\mathfrak{g}) \otimes K) \rightarrow \bigoplus_{i=1}^{k} H^{0}\left(X, K^{d_{i}}\right),
$$

where $d_{i}=\operatorname{deg} p_{d_{i}}$ for $i=1, \ldots, k$. In [13] it is proved that this map is proper and it is well defined uniquely.

We have the following result ([13, Prop. 4.1]).

Proposition 1. Let $E$ be a stable principal $G$-bundle over $X$. Then

$$
\operatorname{dim} H^{0}(X, E(\mathfrak{g}) \otimes K)=\operatorname{dim} \bigoplus_{i=1}^{k} H^{0}\left(X, K^{d_{i}}\right) .
$$

This says that

$$
\operatorname{dim} T_{E}^{*} M_{*}(G)=\operatorname{dim} \bigoplus_{i=1}^{k} H^{0}\left(X, K^{d_{i}}\right),
$$

where $M_{*}(G)$ is the smooth locus of $M(G)$. That is,

$$
\operatorname{dim} M(G)=\operatorname{dim} \bigoplus_{i=1}^{k} H^{0}\left(X, K^{d_{i}}\right)
$$


The map $\mathcal{H}$ is called the Hitchin map and the vector space

$$
B(G)=\bigoplus_{i=1}^{r} H^{0}\left(X, K^{d_{i}}\right)
$$

is called the base of the Hitchin map. In [13] it is proved that $\operatorname{dim} M(G)=$ $\operatorname{dim} B(G)$. Therefore, the Hitchin map induces $n=\operatorname{dim} M(G)$ complex valued functions $f_{1}, \ldots, f_{n}$ on $T_{E}^{*} M_{*}(G)$. Since the invariant homogeneous polynomials act on the Higgs fields of the bundles, it is clear that the functions $f_{i}$ are defined on $T^{*} M_{*}(G)$. In [13, Prop. 4.5], Hitchin proved the following result.

Proposition 2. The $n$ functions $f_{i}$ defined on $T^{*} M_{*}(G)$ Poisson-commute (with the canonical symplectic structure of the cotangent bundle).

This gives a completely integrable system on $M(G)$ (see [2]).

To summarize, the moduli space of principal $G$-bundles, $M(G)$, gives the structure of a Hitchin system, that is, there is a moduli space of $G$-Higgs bundles, $\mathcal{M}(G)$, equipped with a Hitchin map, $\mathcal{H}$, which is proper and induces an integrable system, and a Zariski dense open set of $\mathcal{M}(G), T^{*} M_{*}(G)$, whose complement has codimension $\geq 2$. This Hitchin map is well defined uniquely.

We now recall some facts about spectral curves and Prym varieties associated to the moduli space of $G$-Higgs bundles. Let $\rho$ be an irreducible representation of $G$ of dimension $n$. This representation allows us to see each $G$-Higgs bundle as a pair $(E, \varphi)$ where $E$ is a certain holomorphic vector bundle of rank $n$ and $\varphi \in H^{0}(X, E(\mathfrak{g}) \otimes K)$.

Given an element $a \in B$ of the base of the Hitchin map, it defines a cover $\pi_{a}: X_{a} \rightarrow X$ whose general fiber is given by the roots of a certain equation determined by $a$. More precisely, given any $a \in B$, this element induces a curve $X_{a}$ which is a cover of $X$, is contained in $|K|$ and whose fibers can be identified with the roots of a given equation. This curve is called spectral curve. The construction of this spectral curve is done in detail by Donagi in [8 for any complex reductive Lie group $G$. Also for any reductive complex Lie group $G$, the Prym variety of the curve $X_{a}, \operatorname{Prym}\left(X_{a}\right)$, is defined to be an abelian subvariety of the Jacobian of $X_{a}$ such that the fiber of the Hitchin map, which is a proper map, over $a$ is isomorphic to that variety: if $a \in B$, we have that

$$
\mathcal{H}^{-1}(a) \cong \operatorname{Prym}\left(X_{a}\right) \text {. }
$$

In [2], Adler and Moerbeke raised the question of the dependence of the spectral curve on the representation $\rho$. They proved that two different representations of $G$ may lead to different spectral curves but their Jacobian varieties must contain a common abelian subvariety in which the Prym variety is contained. So the Prym variety is intrinsically defined for every complex reductive Lie group $G$ and also the spectral curves are intrinsically defined when the representation $\rho$ is fixed, which will be our case. is

When $G=F_{4}$ we have that the base of the Hitchin map $\mathcal{H}: T^{*} M_{*}\left(F_{4}\right) \rightarrow B\left(F_{4}\right)$

$$
B\left(F_{4}\right)=H^{0}\left(K^{2}\right) \oplus H^{0}\left(K^{6}\right) \oplus H^{0}\left(K^{8}\right) \oplus H^{0}\left(K^{12}\right) .
$$


Similarly, when $G=E_{6}$, the base of the Hitchin map is

$$
B\left(E_{6}\right)=H^{0}\left(K^{2}\right) \oplus H^{0}\left(K^{5}\right) \oplus H^{0}\left(K^{6}\right) \oplus H^{0}\left(K^{8}\right) \oplus H^{0}\left(K^{9}\right) \oplus H^{0}\left(K^{12}\right) .
$$

If $G=F_{4}$ and $a=\left(a_{2}, a_{6}, a_{8}, a_{12}\right)$ is a general element of the base $B$ of the Hitchin map, where $a_{r} \in H^{0}\left(K^{r}\right)$, the spectral curve $X_{a}$ associated to $a$ is the curve in the total space $|K|$ of $K$ defined by the equation

$$
y^{12}+\sum_{r=2,6,8,12} a_{r}(x) y^{12-r}=0
$$

where $x$ denotes the coordinate for the curve $X$ and $y$ denotes the tautological coordinate $d x$ along the fibers of the projection $|K| \rightarrow X$. Observe that, if $(E, \varphi)$ is an $F_{4}$-Higgs bundle with $\mathcal{H}(E, \varphi)=a$, then the previous equation is a factor of the characteristic polynomial of $\varphi$. Similarly, if $G=E_{6}$, the element $a$ is of the form $a=\left(a_{2}, a_{5}, a_{6}, a_{8}, a_{9}, a_{12}\right)$ and the spectral curve $X_{a}$ is the curve in $|K|$ defined by the equation

$$
y^{12}+\sum_{r=2,5,6,8,9,12} a_{r}(x) y^{12-r}=0 .
$$

From now on, we fix $G=F_{4}$ or $E_{6}$. The fiber of the cotangent bundle $T^{*} M_{*}(G)$ at a simple principal $G$-bundle $E$ can be identified with the group of Higgs fields

$$
T_{E}^{*} M_{*}(G) \cong H^{0}(X, E(\mathfrak{g}) \otimes K) .
$$

It is well known that $T^{*} M_{*}(G)$ admits an intrinsic $\mathbb{C}^{*}$-action which is defined by multiplication on the Higgs field, since the cotangent spaces are $\mathbb{C}$-vector spaces: if $(E, \varphi) \in T^{*} M_{*}(G)$ and $\lambda \in \mathbb{C}^{*}$, then

$$
\lambda \cdot(E, \varphi)=(E, \lambda \varphi) .
$$

We also have a natural action of $\mathbb{C}^{*}$ on the base of the Hitchin map. In the case of $F_{4}$, this action can be formulated in this way: if $\left(a_{2}, a_{6}, a_{8}, a_{12}\right) \in B\left(F_{4}\right)$ and $\lambda \in \mathbb{C}^{*}$, then

$$
\lambda \cdot\left(a_{2}, a_{6}, a_{8}, a_{12}\right)=\left(\lambda^{2} a_{2}, \lambda^{6} a_{6}, \lambda^{8} a_{8}, \lambda^{12} a_{12}\right) .
$$

Similarly, if $\left(a_{2}, a_{5}, a_{6}, a_{8}, a_{9}, a_{12}\right) \in B\left(E_{6}\right)$ and $\lambda \in \mathbb{C}^{*}$, then

$$
\lambda \cdot\left(a_{2}, a_{5}, a_{6}, a_{8}, a_{9}, a_{12}\right)=\left(\lambda^{2} a_{2}, \lambda^{5} a_{5}, \lambda^{6} a_{6}, \lambda^{8} a_{8}, \lambda^{9} a_{9}, \lambda^{12} a_{12}\right) .
$$

Lemma 1. Let $G$ be the complex simple Lie group $F_{4}$ or $E_{6}$ and let $B$ be the vector space $B\left(F_{4}\right)$ or $B\left(E_{6}\right)$, respectively, defined in (5) and (6). Let $\mathcal{H}: T^{*} M_{*}(G) \rightarrow B$ be the Hitchin map. Then, the action of $\mathbb{C}^{*}$ on $B$ defined in (7) or (8), respectively, is the unique $\mathbb{C}^{*}$-action on $B$ which makes the Hitchin map $\mathbb{C}^{*}$-equivariant, with the intrinsic $\mathbb{C}^{*}$-action defined in $T^{*} M_{*}(G)$ by multiplication by complex numbers.

Proof. The cotangent space $T^{*} M_{*}(G)$ is naturally embedded in the moduli space $\mathcal{M}(G)$ of polystable $G$-Higgs bundles and the codimension of the embedding is at least two in the smooth fibers $([10])$. Since the Hitchin map $\mathcal{H}$ extends to a map $\overline{\mathcal{H}}: \mathcal{M}(G) \rightarrow B$, the result holds for $T^{*} M_{*}(G)$. 
Lemma 2. Let $G$ be the complex simple Lie group $F_{4}$ or $E_{6}$ and let $B$ be the vector space $B\left(F_{4}\right)$ or $B\left(E_{6}\right)$, respectively, defined in (5) and (6). Let $\mathcal{H}: T^{*} M_{*}(G) \rightarrow B$ be the Hitchin map. Let $B_{r}$ be the summand $H^{0}\left(K^{r}\right)$ of $B$, for $r=2,5,6,8,9,12$ or $r=2,6,8,12$. Then, $B_{r}$ is uniquely determined by the map $\mathcal{H}$. In particular, $B_{12}$ is intrinsically defined. The linear structure of $B$ can also be recovered from $\mathcal{H}$.

Proof. The action of $\mathbb{C}^{*}$ on $B$ defined in (7) or $(8)$ determines the origin $0 \in B$, since it is the only fixed point of the action. The vector space $B$ is thus endowed with an affine structure and, moreover, one can recover the tangent subspace $T_{0} B_{r}$ as the subspace of $T_{0} B$ where the weight of the $\mathbb{C}^{*}$-action is $\lambda^{r}$. We consider the pull-back of this space $T_{0} B_{r}$ to $T_{E}^{*} M_{*}(G)$, where $E$ is a fixed polystable principal $G$-bundle. The space $B_{r}$ can be recovered as the projection over $B$ of the induced linear space. This also allows us to recover the linear structure of $B$.

Finally, the space $B_{12}$ is then intrinsically defined since the weight spaces of $B$ are well-defined, the action of $\mathbb{C}^{*}$ on $T^{*} M_{*}(G)$ is intrinsically defined and, by Lemma 1, the induced action of $\mathbb{C}^{*}$ on $B$ is uniquely determined.

\section{TORELLI THEOREM FOR PRINCIPAL $F_{4}$ AND $E_{6}$-BUNDLES}

Let $X$ be a smooth complex projective irreducible curve of genus $g \geq 3$. Let $G$ be the complex simple Lie group $F_{4}$ or $E_{6}$ and let $M(G)$ be the moduli space of principal $G$-bundles. Let $\mathcal{H}$ be the Hitchin map defined in (4) and let $B$ be its base. From (5) and (6) we know that

$$
B=H^{0}\left(K^{2}\right) \oplus H^{0}\left(K^{6}\right) \oplus H^{0}\left(K^{8}\right) \oplus H^{0}\left(K^{12}\right)
$$

when $G=F_{4}$ and

$$
B=H^{0}\left(K^{2}\right) \oplus H^{0}\left(K^{5}\right) \oplus H^{0}\left(K^{6}\right) \oplus H^{0}\left(K^{8}\right) \oplus H^{0}\left(K^{9}\right) \oplus H^{0}\left(K^{12}\right)
$$

when $G=E_{6}$. Let $B_{12}$ be the component $H^{0}\left(K^{12}\right)$ of $B$ in the expressions above, which is well defined by Lemma 2 .

In this section we will prove the Torelli theorem for $M(G)$, that is, we will see that the moduli space determines the curve up to isomorphisms.

Let $R \subseteq B$ be the divisor given by invariant polynomials corresponding to singular spectral curves. The divisor $R$ consists of two components, $R=R_{1} \cup R_{2}$, where $R_{1}$ consists of those elements whose component in $B_{12}$ has a double root and $R_{2}$ consists of elements which correspond to polynomials which have a node. Let $R_{i}^{0}$ be the locus of $R_{i}$, for $i=1,2$, consisting of points in $B$ whose corresponding spectral curves contain no extra singularities.

We have to prove that $R$ is intrinsically defined. To do that we will see that the singular spectral curves in the Hitchin total space correspond with points in the Hitchin base, $a \in B$, such that the fiber of $a$ by the Hitchin map, which is isomorphic to $\operatorname{Prym}\left(X_{a}\right)$, is a rational variety and that the nonsingular curves correspond to points in $B$ whose associated Prym variety is an abelian variety. Since the condition of being a rational variety is preserved by isomorphisms of the Hitchin total space, $R$ will be intrinsically defined.

This follows from the discussion before Proposition 3.1 in [5], which we particularize here to our groups. We briefly sketch the construction explained there 
for the symplectic group. Let $a \in B$ be a point in the base of the Hitchin map such that the corresponding spectral curve, $X_{a}$, admits only one singularity at a point $r$, which is a simple node. Let $\pi_{a}: \widetilde{X}_{a} \rightarrow X_{a}$ be its normalization and let $r_{1}, r_{2}$ be the preimages of $r$ in $\widetilde{X}_{a}$. We consider the compactified Jacobian of $X_{a}$, $\bar{J}\left(X_{a}\right)$, which parametrizes torsionfree sheaves of rank 1 and degree 0 on $X_{a}$. It is birational to a $\mathbb{P}^{1}$-fibration over $J\left(\widetilde{X_{a}}\right)$, which we will call $P$. A point in $P$ can be seen as a line bundle $L$ over $\widetilde{X_{a}}$ together with a quotient $q: L_{r_{1}} \oplus L_{r_{2}} \rightarrow \mathbb{C}$, up to scalar multiple. Since $X_{a}$ comes with an automorphism of order three, $\tau$, this induces an automorphism of order three of $\widetilde{X_{a}}, \widetilde{\tau}$. This automorphism acts on any pair $(L, q)$ as before in the following way: $\widetilde{\tau}(L, q)=\left(\left(\tau^{2}\right)^{*} L^{*} \otimes \tau^{*} L^{*}, q^{\tau}\right)$, where $q^{\tau}$ is represented by $[c: a: b]$ if $q$ is represented by $[a: b: c]$ (with the notation of [5]). Now, the automorphism $\widetilde{\tau}$ induces an automorphism of order three on $\bar{J}\left(X_{a}\right)$ whose restriction to the open subset $J\left(X_{a}\right)$ of $\bar{J}\left(X_{a}\right)$ is given by $L \mapsto\left(\tau^{2}\right)^{*} L \otimes \tau^{*} L^{*}$. Then the fixed point variety of this automorphism is

$$
\left\{L \in \bar{J}\left(X_{a}\right):\left(\tau^{2}\right)^{*} L \otimes \tau^{*} L \otimes L \cong \mathcal{O}\right\} .
$$

We will call this variety $\operatorname{Prym}\left(X_{a}, \tau\right)$. This is a uniruled variety, since it admits a surjective morphism from a subvariety of $P$, which is a $\mathbb{P}^{1}$-fibration, defined by pull-back of $P \rightarrow J\left(\widetilde{X_{a}}\right)$.

Now, if $X_{a}$ is a spectral curve which admits three simple nodes and, as before, $\widetilde{X_{a}}$ denotes its normalization, then $\bar{J}\left(X_{a}\right)$ is birational to a $\mathbb{P}^{1} \times \mathbb{P}^{1} \times \mathbb{P}^{1}$-bundle $P$ on $J\left(\widetilde{X_{a}}\right)$, the spectral curve $X_{a}$ admits an automorphism of order three, $\tau$, which permutes the three nodes and this automorphism induces an automorphism of order three of $P$ such that the fixed points admit the same form as before, so this defines a $\mathbb{P}^{1}$-fibration on $\operatorname{Prym}\left(\widetilde{X_{a}}\right)$. The image on $\bar{J}\left(X_{a}\right)$ is exactly $\operatorname{Prym}\left(X_{a}, \tau\right)$, which is a uniruled variety.

From this construction, we may prove Propositions 3 , 4 and 5 These are analogous to Propositions 3.1 and 3.2 and Theorem 3.3 of [5], with the only special features of the construction sketched before, and the same proofs work for our purpose. We write them here for the paper to be self-contained.

Proposition 3. Let $\mathcal{H}: T^{*} M(G) \rightarrow B$ be the Hitchin map defined in (4) and let $a \in B$. Then if $a \notin R$, the fiber $\mathcal{H}^{-1}(a)$ is an open subset of an abelian variety. Also, if $a \in R_{1}^{0} \cup R_{2}^{0}$, then the fiber $\mathcal{H}^{-1}(a)$ is an open subset of the uniruled variety $\operatorname{Prym}\left(X_{a}, \tau\right)$ defined in $(9)$. In both cases, the complement of the open subsets is of codimension at least 2 .

Proof. Let $\mathcal{M}(G)$ be the moduli space of $G$-Higgs bundles, where $G$ is the group under consideration. Let $\overline{\mathcal{H}}: \mathcal{M}(G) \rightarrow B$ be the Hitchin map defined on $\mathcal{M}(G)$. Since the Hitchin map $\overline{\mathcal{H}}$ is proper, if $a \in B \backslash R$, the corresponding fiber by the Hitchin map, $\overline{\mathcal{H}}^{-1}(a)$, is an abelian variety. From [10, Theorem II.6], it follows that the complement $\mathcal{M}(G) \backslash T^{*} M(G)$ is of codimension $\geq 3$, under the assumption that $g \geq 3$. Therefore, the intersection of this complement with $R_{i}$ is of codimension at least 2 in $R_{i}$ for $i=1,2$, so this is also true for the codimension of the corresponding 
intersections with the fiber of $a$ by the Hitchin map, defined on $\mathcal{M}(G)$ and $T^{*} M(G)$, $\overline{\mathcal{H}}^{-1}(a) \backslash \mathcal{H}^{-1}(a) \subseteq \overline{\mathcal{H}}^{-1}(a)$, when $a \in R_{1}^{0} \cup R_{2}^{0}$.

Proposition 4. Let $\mathcal{H}: T^{*} M(G) \rightarrow B$ be the Hitchin map defined in (4). The hypersurfaces $\mathcal{H}^{-1}\left(R_{i}\right)$, for $i=1,2$, are irreducible.

Proof. Let $\overline{\mathcal{H}}: \mathcal{M}(G) \rightarrow B$ be the Hitchin map defined on $\mathcal{M}(G)$. From [10, Theorem II.5], the fibers of the Hitchin map $\overline{\mathcal{H}}$ are Lagrangian, hence of half dimension. This implies that the codimension of $\mathcal{H}^{-1}\left(R \backslash R_{1}^{0} \cup R_{2}^{0}\right)$ is that of $R \backslash R_{1}^{0} \cup R_{2}^{0}$ in $B$, which is at least two, since the fibers of $\mathcal{H}$ are equidimensional. This proves the result.

Proposition 5. Let $\mathcal{H}: T^{*} M(G) \rightarrow B$ be the Hitchin map defined in (4). The subvariety $\mathcal{H}^{-1}(R)$ is the closure of the union of the complete rational curves in $T^{*} M(G)$.

Proof. The image by $\mathcal{H}$ of any $\mathbb{P}^{1}$ contained in $\mathcal{H}^{-1}(R)$ must be a point of $B$, since it is a complete curve, so this $\mathbb{P}^{1}$ is contained in a fiber of the Hitchin map over an element $a \in B$. This $a$ must be in $R$, by Proposition 3 , so this $\mathbb{P}^{1}$ is contained in $\mathcal{H}^{-1}(R)$. Moreover, from Proposition 3 there exists a family of $\mathbb{P}^{1}$ covering the fibers over the elements of $R_{1}^{0} \cup R_{2}^{0}$. The closure of this family is $\mathcal{H}^{-1}(R)$ because this variety is irreducible, by Proposition 4 This proves the result.

We may now define the following divisor

$$
\mathcal{C}=B_{12} \cap\left(R_{1} \cup R_{2}\right) .
$$

The divisor $\mathcal{C}$ is intrinsically defined, since $R$ is intrinsically defined and $B_{12}$ is uniquelly determined by $\mathcal{H}$, by Lemma 2

Lemma 3. The divisor $\mathcal{C}$ defined in $(10)$ is irreducible.

Proof. If $a_{12} \in B_{12}$, the corresponding element in $B$ is $\left(0, \ldots, 0, a_{12}\right)$ and the induced polynomial is $y^{12}+a_{12}(x)=0$. The corresponding spectral curve has $(x, 0)$ as a singular point if and only if $a_{12}(x)=a_{12}^{\prime}(x)=0$. Therefore, $\mathcal{C}=R_{1} \cap B_{12}$. This proves the result.

Proposition 6. Let $\mathcal{C}$ be the divisor defined in 10 . Then, $\mathbb{P C} \subseteq \mathbb{P} B_{12}$ is the dual variety of $X \subseteq \mathbb{P} B_{12}^{*}$ for the embedding given by the linear system $\left|K^{12}\right|$.

Proof. Any element $a_{12} \in B_{12}$ comes from an element $\left(0, \ldots, 0, a_{12}\right) \in B$ and, then, from a curve of the form $y^{12}+a_{12}(x)=0$. We have that $a_{12} \in \mathcal{C}$ if and only if there exists $x \in X$ such that $a_{12}(x)=a_{12}^{\prime}(x)=0$. This implies that

$$
a_{12} \in H^{0}\left(K^{12}(-2 x)\right) \subseteq H^{0}\left(K^{12}\right) .
$$

From this, it is clear that

$$
\mathbb{P C}=\cup_{x \in X} \mathbb{P} H^{0}\left(K^{12}(-2 x)\right) \subseteq \mathbb{P} H^{0}\left(K^{12}\right)=\mathbb{P} B_{12} .
$$

Observe now that $\left|K^{12}\right|$ is very ample, since

$$
\operatorname{deg} K^{12}=12(2 g-2)>2 g+1,
$$

so the result finally holds. 
Theorem 1. Let $X$ and $X^{\prime}$ be smooth projective curves of genus $g$ and $g^{\prime}$, respectively, with $g, g^{\prime} \geq 3$. Let $G$ be the simple complex Lie group $F_{4}$ or $E_{6}$ and let $M_{*}(G)$ and $M_{*}^{\prime}(G)$ be the moduli spaces of stable and simple principal $G$-bundles over $X$ and $X^{\prime}$, respectively. Suppose that $M_{*}(G) \cong M_{*}^{\prime}(G)$. Then, $X \cong X^{\prime}$.

Proof. Suppose that $M_{*}(G) \cong M_{*}^{\prime}(G)$ and let $F: M_{*}(G) \rightarrow M_{*}^{\prime}(G)$ be an isomorphism. Then, $d F^{*}$ is an isomorphism at the level of cotangent bundles, $d F^{*}$ : $T^{*} M_{*}(G) \rightarrow T^{*} M_{*}^{\prime}(G)$. Let $B$ and $B^{\prime}$ be the bases of the Hitchin map of $M_{*}(G)$ and $M_{*}^{\prime}(G)$, respectively. Let $B_{12}$ and $B_{12}^{\prime}$ be the subspaces of $B$ and $B^{\prime}$ univocally determined in Lemma 2. In both subspaces we have an action of $\mathbb{C}^{*}$ which is the restriction to that subspace of the action defined in (7) and (8): if $\lambda \in \mathbb{C}^{*}, \lambda$ acts on $B_{12}$ and $B_{12}^{\prime}$ by multiplication by $\lambda^{12}$.

It is also clear that the isomorphism $d F$ induces an isomorphism $f: B \rightarrow B^{\prime}$ such that the following diagram is commutative:

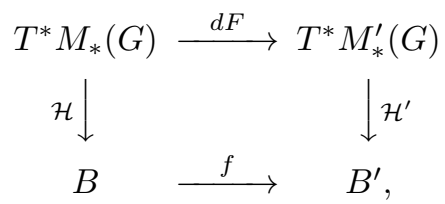

where $\mathcal{H}: T^{*} M_{*}(G) \rightarrow B$ and $\mathcal{H}^{\prime}: T^{*} M_{*}^{\prime}(G) \rightarrow B^{\prime}$ are the Hitchin maps. It is clear from the definition of the actions and from Lemma 1 that $f$ is $\mathbb{C}^{*}$-equivariant. This shows that $f$ restricts to a linear isomorphism $f_{12}: B_{12} \rightarrow B_{12}^{\prime}$, which gives rise to an isomorphism

$$
\bar{f}_{12}: \mathbb{P} B_{12} \rightarrow \mathbb{P} B_{12}^{\prime} .
$$

Then, an isomorphism of the dual varieties is induced. From Proposition 6 we get $X \cong X^{\prime}$.

This theorem gives a Torelli type theorem when we consider the open subvariety of stable and simple principal $G$-bundles. Since this open subset is dense in $M(G)$, we conclude the desired Torelli theorem.

Theorem 2. Let $X$ and $X^{\prime}$ be smooth projective curves of genus $g$ and $g^{\prime}$, respectively, with $g, g^{\prime} \geq 3$. Let $G$ be the complex simple Lie group $F_{4}$ or $E_{6}$ and let $M(G)$ and $M^{\prime}(G)$ be the moduli spaces of principal $G$-bundles over $X$ and $X^{\prime}$, respectively. Suppose that $M(G) \cong M^{\prime}(G)$. Then, $X \cong X^{\prime}$.

Proof. Suppose that $M_{*}(G) \cong M_{*}^{\prime}(G)$ and let $F: M(G) \rightarrow M^{\prime}(G)$ be an isomorphism. This isomorphism restricts to an isomorphism of the smooth locus of $M(G)$ to the smooth locus of $M^{\prime}(G)$. By Theorem 1 the result holds.

\section{Automorphisms of $M\left(F_{4}\right)$}

Let $X$ be a smooth complex projective irreducible curve of genus $g \geq 3$ and let $M\left(F_{4}\right)$ be the moduli space of polystable principal $F_{4}$-bundles over $X$. Observe that any automorphism of the base curve $X, \alpha: X \rightarrow X$, induces an automorphism of the moduli space $M\left(F_{4}\right)$ by taking the pull-back

$$
\alpha^{*}: M\left(F_{4}\right) \rightarrow M\left(F_{4}\right), \quad E \mapsto \alpha^{*} E .
$$


The aim of this section is to prove that $M\left(F_{4}\right)$ admits no other automorphisms that those coming from automorphisms of the curve $X$ as defined in (11). The strategy will be the following. Any automorphism $\Phi$ of $M\left(F_{4}\right)$ induces an automorphism of the curve $X, \alpha$, which is given by the Torelli theorem (Theorem 2). We will prove that $\Phi=\alpha^{*}$, with the notation of (11). We may suppose that $\alpha$ is the identity and it suffices to show that $\Phi$ itself is the identity. To prove this we will see that, in this situation, for any stable and simple principal $F_{4}$-bundle $E$, the adjoint vector bundles $E\left(\mathfrak{f}_{4}\right)$ and $\Phi(E)\left(\mathfrak{f}_{4}\right)$ are isomorphic with their structures of Lie algebra bundles and that, in this case, it must be $E \cong \Phi(E)$ as principal $F_{4}$-bundles. The final result (Theorem 3 follows from the fact that the open subset of stable and simple principal $F_{4}$-bundles is dense in $M\left(F_{4}\right)$.

Lemma 4. Let $E$ be a simple $F_{4}$-bundle and let $x \in X$ be any point. Then $H^{0}\left(E\left(\mathfrak{f}_{4}\right)(x)\right)=0$.

Proof. Any section $s \in H^{0}\left(E\left(\mathfrak{f}_{4}\right)(x)\right)$ can be seen as a vector bundle endomorphism of $E$ which is compatible with the forms that define the $F_{4}$-structure in $E$ and satisfying $s(x)=0$. Since $E$ is simple by hypothesis, it should be $s=0$.

Lemma 5. Let $B$ be the base of the Hitchin map of $M\left(F_{4}\right)$ defined in (5) and let $B_{12}$ be the subspace of $B$ given by Lemma 2. Let $E$ be a simple principal $F_{4}$-bundle over $X$. Let $\mathcal{H}_{12}: H^{0}\left(E\left(\mathfrak{f}_{4}\right) \otimes K\right) \rightarrow B_{12}$ be the composition of the Hitchin map $\mathcal{H}$ with the projection of $B$ over $B_{12}$ (this projection exists since the summands and the linear structure of the base $B$ are uniquely determined by Lemma 2). For each $x \in X$, let

$$
B_{12, x}=H^{0}\left(K^{12}(-x)\right) \subseteq B_{12} .
$$

Then

$H^{0}\left(E\left(\mathfrak{f}_{4}\right) \otimes K(-x)\right)=\left\{s \in H^{0}\left(E\left(\mathfrak{f}_{4}\right) \otimes K\right): \mathcal{H}_{12}(s+t) \in B_{12, x} \forall t \in \mathcal{H}^{-1}\left(B_{12, x}\right)\right\}$.

Proof. Observe first that the subset $B_{12, x}$ is intrinsically defined since the Hitchin map $\mathcal{H}$ and $B_{12}$ are uniquely determined: $B_{12, x}$ is nothing but the image by the Hitchin map of those bundles $(E, \varphi)$ such that $p_{12}(\varphi(x))=0$. The exact sequence of sheaves

$$
\left.0 \rightarrow E\left(\mathfrak{f}_{4}\right) \otimes K(-x) \rightarrow E\left(\mathfrak{f}_{4}\right) \otimes K \rightarrow E\left(\mathfrak{f}_{4}\right) \otimes K\right|_{x} \rightarrow 0
$$

induces an exact sequence of cohomology sets of the form

$$
\left.H^{0}\left(E\left(\mathfrak{f}_{4}\right) \otimes K\right) \rightarrow E\left(\mathfrak{f}_{4}\right) \otimes K\right|_{x} \rightarrow H^{1}\left(E\left(\mathfrak{f}_{4}\right) \otimes K(-x)\right) .
$$

From Lemma 4 and Serre duality we obtain that

$$
H^{1}\left(E\left(\mathfrak{f}_{4}\right) \otimes K(-x)\right)=H^{0}\left(E\left(\mathfrak{f}_{4}\right)(x)\right)^{*}=0
$$

so the map $\left.H^{0}\left(E\left(\mathfrak{f}_{4}\right) \otimes K\right) \rightarrow E\left(\mathfrak{f}_{4}\right) \otimes K\right|_{x}$, which consists in evaluating in $x$, is surjective.

Take now $s \in H^{0}\left(E\left(\mathfrak{f}_{4}\right) \otimes K\right)$. It is clear that $\mathcal{H}_{12}(s) \in B_{12}$ if and only if $p_{12}(s(x))=0$, where $p_{12}$ is the fundamental homogeneous invariant polynomial with degree 12. The result then follows from the following easy linear algebra fact: the only possibility for such an element $s$ which satisfies $p_{12}(s(x)+t(x))=0$ for 
all $t$ with $p_{12}(t(x))=0$ is $s(x)=0$ (observe that if a matrix $A \in \mathfrak{f}_{4}$ satisfies that $p_{12}(A+B)=0$ for any $B \in \mathfrak{f}_{4}$ with $p_{12}(B)=0$, then, for any such $B$, the whole line through $A$ and $n B, n \in \mathbb{N}$ is contained in the zero set of the polynomial $p_{12}$; this necessarily implies $A=0$ ).

From Lemma 5, we may define a bundle $\mathcal{F}$ over the curve $X$, associated to each simple principal $F_{4}$-bundle $E$, whose fiber over any point $x \in X$ is

$$
\mathcal{F}_{x}=H^{0}\left(E\left(\mathfrak{f}_{4}\right) \otimes K(-x)\right) .
$$

This bundle $\mathcal{F}$ is nothing but the kernel of the homomorphism of vector bundles

$$
H^{0}\left(E\left(\mathfrak{f}_{4}\right) \otimes K\right) \otimes \mathcal{O} \rightarrow E\left(\mathfrak{f}_{4}\right) \otimes K,
$$

where $\mathcal{O} \rightarrow X$ denotes the trivial line bundle.

Theorem 3. Let $M\left(F_{4}\right)$ be the moduli space of polystable principal $F_{4}$-bundles over the complex algebraic curve $X$. Let $\Phi: M\left(F_{4}\right) \rightarrow M\left(F_{4}\right)$ be an automorphism of $M\left(F_{4}\right)$. Then there exists an automorphism of $X, \alpha$, such that $\Phi=\alpha^{*}$, where $\alpha^{*}$ is defined in (11).

Proof. Observe first that we may suppose that the automorphism of $X$ induced by $\Phi$, given by Theorem 2 is the identity.

Let $E$ be a stable and simple principal $F_{4}$-bundle, $\mathcal{H}: H^{0}\left(E\left(\mathfrak{f}_{4}\right) \otimes K\right) \rightarrow B$ be the Hitchin map, and $B$ be the base of the Hitchin map. For $r=2,6,8,12$, let $B_{r}$ be the summand $B_{r}=H^{0}\left(K^{r}\right)$ in the decomposition of $B$, which is well defined by Lemma 2, For each $r=2,6,8,12$, let $\mathcal{H}_{r}$ be the composition of the Hitchin map $\mathcal{H}$ with the projection map of $B$ over $B_{r}$. Let $f: B \rightarrow B$ be the automorphism of $B$ induced by $\Phi$. Let $x \in X$. The arguments of Lemma 2 show that $\mathcal{H}_{r}\left(H^{0}\left(E\left(\mathfrak{f}_{4}\right) \otimes K(-x)\right)\right)$ is preserved by $f$, so $d \Phi^{*}$ preserves

$$
\bigcap_{r=2,6,8,12} \mathcal{H}_{r}^{-1}\left(\mathcal{H}_{r}\left(H^{0}\left(E\left(\mathfrak{f}_{4}\right) \otimes K(-x)\right)\right)\right) .
$$

The image of this set under the surjective map $\left.H^{0}\left(E\left(\mathfrak{f}_{4}\right) \otimes K\right) \rightarrow E\left(\mathfrak{f}_{4}\right) \otimes K\right|_{x}$ coincides with the nilpotent cone

$$
N(E)_{x}=\left\{\left.A \in E\left(\mathfrak{f}_{4}\right) \otimes K\right|_{x}: A \text { is nilpotent }\right\} .
$$

Observe that the union of all the nilpotent cones when $x$ goes through $X$ gives the nilpotent bundle over $X$ associated to $E$, which we call $N(E)$. The previous discussion shows that we have an isomorphism of the nilpotent bundles $N(E) \rightarrow$ $N(\Phi(E)$ ) as bundles over $X$ (since the automorphism of the curve $X$ induced by $\Phi$ is the identity, as we have supposed).

The main result in 9] shows that this automorphism between the nilpotent cone bundles induces an automorphism of the corresponding Borel flag varieties (which parametrizes the flags induced in the $F_{4}$-bundle by the reductions of structure group to the Borel subgroup of $F_{4}$ ), which we will call Flag $(E)$ and $\operatorname{Flag}(\Phi(E))$.

The automorphism $\operatorname{Flag}(E) \rightarrow \operatorname{Flag}(\Phi(E))$ induces a Lie algebra bundle isomorphism $E\left(\mathfrak{f}_{4}\right) \cong \Phi(E)\left(\mathfrak{f}_{4}\right)$. Observe that giving a Lie algebra bundle structure is 
equivalent to giving a principal $\operatorname{Aut}\left(\mathfrak{f}_{4}\right)$-bundle together with a reduction of structure group to $F_{4}$, corresponding to $E$. Since $F_{4}$ is centerless and it admits no outer automorphisms, the Lie algebra automorphism $E\left(\mathfrak{f}_{4}\right) \cong \Phi(E)\left(\mathfrak{f}_{4}\right)$ gives an automorphism of principal $F_{4}$-bundles $E \cong \Phi(E)$.

This holds for any $E$ of a dense open subset of $M\left(F_{4}\right)$, so the result is finally proved.

\section{Automorphisms of $M\left(E_{6}\right)$}

Let $M\left(E_{6}\right)$ be the moduli space of polystable principal $E_{6}$-bundles over the complex algebraic curve of genus $g \geq 3, X$. At first glance, we recognize three types of automorphisms of $M\left(E_{6}\right)$ : one coming from the action of $\operatorname{Out}\left(E_{6}\right)$ on $M\left(E_{6}\right)$, those induced by the elements of the group $H^{1}\left(X, Z\left(E_{6}\right)\right)$, and those given as the pull-back by an automorphism of the base curve.

(A) There is an automorphism of $M\left(E_{6}\right)$ coming from the action of $\operatorname{Out}\left(E_{6}\right) \cong$ $\mathbb{Z}_{2}$.

In [3], we decribe the action of $\operatorname{Out}(G)$, for a semisimple complex Lie group $G$, on the moduli space, $M(G)$, of polystable principal $G$-bundles, which we recall here: if $E \in M(G)$ and $\rho \in \operatorname{Out}(G)$, the $G$-bundle $\rho(E)$ is defined to have the same total space as $E$ and equipped with the action of $G$ given by

$$
e \star g=e \rho(g)^{-1},
$$

for $g \in G$. In our case, let $\sigma$ be the outer involution of $E_{6}$. The induced automorphism of $M\left(E_{6}\right)$, which we also denote by $\sigma$, is

$$
\sigma: M\left(E_{6}\right) \rightarrow M\left(E_{6}\right), \quad \sigma(E)=E^{*} .
$$

This is of course a non trivial involution of $M\left(E_{6}\right)$, since every fixed point of $\sigma$ is strictly polystable (see [3, Proposition 7.2]).

(B) The group of automorphisms of the base curve, $\operatorname{Aut}(X)$, acts on $M\left(E_{6}\right)$.

The action of $\operatorname{Aut}(X)$ on $M\left(E_{6}\right)$ is defined by taking the pull-back of the bundle: if $E \in M\left(E_{6}\right)$ and $\alpha \in \operatorname{Aut}(X)$, we define

$$
\alpha^{*}: M\left(E_{6}\right) \rightarrow M\left(E_{6}\right), \quad \alpha^{*}(E)=\alpha^{*} E .
$$

By taking the pull-back, the bundle $\alpha^{*} E$ comes with a nondegenerate symmetric trilinear form inherited from $E$. Therefore, $\alpha^{*} E$ admits an $E_{6}$-structure. Observe also that the reductions of $\alpha^{*} E$ to a parabolic subgroup $P$ of $E_{6}$ correspond univocally, via $\alpha$, to the reductions of structure group of $E$ to $P$. From this, it is clear that $\alpha^{*} E$ is polystable (resp. stable) if and only if $E$ is polystable (resp. stable).

(C) The group $H^{1}\left(X, Z\left(E_{6}\right)\right) \cong H^{1}\left(X, \mathbb{Z}_{3}\right)$ acts on $M\left(E_{6}\right)$.

If $Z\left(E_{6}\right)$ denotes the center of $E_{6}, Z\left(E_{6}\right) \cong \mathbb{Z}_{3}$, the group of isomorphism classes of principal $Z\left(E_{6}\right)$-bundles, $H^{1}\left(X, Z\left(E_{6}\right)\right)$, also acts on $M\left(E_{6}\right)$. Let $E$ be a principal $E_{6}$-bundle with projection map $\pi_{E}: E \rightarrow X$ and $L \in H^{1}\left(X, Z\left(E_{6}\right)\right)$ a principal $Z\left(E_{6}\right)$-bundle with projection $\pi_{L}: L \rightarrow X$. The variety

$$
E \times_{X} L=\left\{(e, l) \in E \times L: \pi_{E}(e)=\pi_{L}(l)\right\}
$$


has a natural structure of principal $E_{6} \times Z\left(E_{6}\right)$-bundle and a projection map $E \times_{X} L$ $\rightarrow X$ induced by $\pi_{E}$ and $\pi_{L}$. In $E \times_{X} L$ we define the following relation $\sim$ : if $\left(e_{1}, l_{1}\right),\left(e_{2}, l_{2}\right) \in E \times_{X} L$, we say that $\left(e_{1}, l_{1}\right) \sim\left(e_{2}, l_{2}\right)$ if there exists an element $\lambda \in Z\left(E_{6}\right)$ such that $\left(e_{2}, l_{2}\right)=\left(e_{1} \lambda, l_{1} \lambda^{-1}\right)$. It is easily checked that this is an equivalence relation and that any two elements of $E \times_{X} L$ which are in the same equivalence class lie on the same point of $X$ by the projection map $E \times_{X} L \rightarrow X$. We define $E \otimes L$ to be the quotient of $E \times_{X} L$ by this equivalence relation,

$$
E \otimes L=E \times_{X} L / \sim .
$$

It is easily seen that there is an induced action of $E_{6}$ on $E \otimes L$ : if $(e, l) \in E \times_{X} L$, $[e, l]$ denotes the class of $(e, l)$ in $E \otimes L$ and $g \in E_{6}$, then $[e, l] g=[e g, l]$. We also have that the projection $E \times_{X} L \rightarrow X$ factors through $E \otimes L$ and that the action of $E_{6}$ on $E \otimes L$ is compatible with the induced projection. This implies that $E \otimes L$ inherits an structure of principal $E_{6}$-bundle over $X$.

It is easy to see that, if $\left\{\varphi_{i j}\right\}$ are the transition functions of $E$ associated to certain open covering of $X$, and $\left\{\psi_{i j}\right\}$ are the transition functions of $L$ associated to the same covering, then $\left\{\psi_{i j} \varphi_{i j}\right\}$ are transition functions of $E \otimes L$. This observation shows that this is a good definition and the map $(L, E) \mapsto E \otimes L$ is an action: if $L_{1}, L_{2} \in H^{1}\left(X, Z\left(E_{6}\right)\right)$ have transition functions $\psi_{i j}$ and $\theta_{i j}$, then $E \otimes\left(L_{1} \otimes L_{2}\right)$ has transition functions $\varphi_{i j} \psi_{i j} \theta_{i j}$, the same as $\left(E \otimes L_{1}\right) \otimes L_{2}$. Similarly, $L^{-1}$ (with transition functions $\left.\psi_{i j}^{-1}\right)$ is the inverse element of $L$ for this action.

We then define the following action of $H^{1}\left(X, Z\left(E_{6}\right)\right)$ on the set of isomorphism classes of principal $E_{6}$-bundles: if $E$ is an $E_{6}$-bundle and $L \in H^{1}\left(X, Z\left(E_{6}\right)\right)$, then we define

$$
L \cdot E=E \otimes L .
$$

It is clear that the isotropic subbundles of such a bundle $E \otimes L$ coincide with the isotropic subbundles of $E$ tensored by $L$ and the tensorization preserves the degrees. Then, $E \otimes L$ is polystable (resp. stable) if and only if $E$ is polystable (resp. stable). Therefore, this defines an action on $M\left(E_{6}\right)$.

We shall prove that this action is effective.

Lemma 6. The action of $H^{1}\left(X, Z\left(E_{6}\right)\right)$ on $M\left(E_{6}\right)$ defined in 12$)$ is effective.

Proof. Let $L \in H^{1}\left(X, Z\left(E_{6}\right)\right)$. Let $E$ be a strictly polystable principal $E_{6}$ bundle such that $E \otimes L$. It suffices to show that $L \cong \mathcal{O}$, the trivial bundle over $X$.

Since $E$ is strictly polystable, from Definition 1 , we may suppose that there exist vector bundles $E_{1}$ and $E_{2}$ of degree zero such that $E_{1}$ is isotropic and $E \cong E_{1} \oplus E_{2}$. It is clear that, in this situation, $E_{1} \cong E_{1} \otimes L$. Let $r$ be the rank of $E_{1}$. Then,

$$
\mathcal{O} \cong \operatorname{det} E \cong \operatorname{det} E \otimes L \cong L^{r} \text {. }
$$

One can always choose $E$ such that $r$ is not multiple of 3. Take, for example, any stable principal $F_{4}$-bundle, $F$, over $X$. The image of $F$ in $M\left(E_{6}\right)$ via the natural map $M\left(F_{4}\right) \rightarrow M\left(E_{6}\right)$ may be decomposed as a direct sum of the form $\mathcal{O} \oplus F$, so in this case we have $r=1$. In a situation like this, the only possibility is $L \cong \mathcal{O}$, as we wanted to see. 
The aim of this section is to prove that $M\left(E_{6}\right)$ admits no other automorphisms than those which can be expressed as combinations of the automorphisms described above. The strategy is the same followed with $F_{4}$ in the previous section. The following auxiliary results (Lemmas 7 and 8) are analogous to Lemmas 4 and 5. respectively, and the same proofs work.

Lemma 7. Let $E$ be a simple $E_{6}$-bundle and let $x \in X$ be any point. Then $H^{0}\left(E\left(\mathfrak{e}_{6}\right)(x)\right)=0$.

Lemma 8. Let $B$ be the base of the Hitchin map of $M\left(E_{6}\right)$ defined in $(6)$ and let $B_{12}$ be the subspace of $B$ given by Lemma 2. Let $E$ be a simple principal $E_{6}$-bundle over $X$. Let $\mathcal{H}_{12}: H^{0}\left(E\left(\mathfrak{e}_{6}\right) \otimes K\right) \rightarrow B_{12}$ be the composition of the Hitchin map $\mathcal{H}$ with the projection of $B$ over $B_{12}$ (this projection exists since the summands and the linear structure of the base $B$ are uniquely determined by Lemma 2). For each $x \in X$, let

$$
B_{12, x}=H^{0}\left(K^{12}(-x)\right) \subseteq B_{12} .
$$

Then

$H^{0}\left(E\left(\mathfrak{e}_{6}\right) \otimes K(-x)\right)=\left\{s \in H^{0}\left(E\left(\mathfrak{e}_{6}\right) \otimes K\right): \mathcal{H}_{12}(s+t) \in B_{12, x} \forall t \in \mathcal{H}^{-1}\left(B_{12, x}\right)\right\}$.

Proposition 7. Let $E$ and $E^{\prime}$ be simple principal $E_{6}$-bundles over $X$ such that $E\left(\mathfrak{e}_{6}\right)$ and $E^{\prime}\left(\mathfrak{e}_{6}\right)$ are isomorphic as Lie algebra bundles via an automorphism $\alpha$ : $E\left(\mathfrak{e}_{6}\right) \rightarrow E^{\prime}\left(\mathfrak{e}_{6}\right)$. Then there exists $L \in H^{1}\left(X, \mathbb{Z}_{3}\right)$ such that $E^{\prime} \cong E \otimes L$ or $E^{\prime} \cong \sigma(E) \otimes L$, where $\sigma \in \operatorname{Out}\left(E_{6}\right)$ is the outer involution of $E_{6}$.

Proof. Giving a Lie algebra bundle structure $E\left(\mathfrak{e}_{6}\right)$ is the same as giving a principal $\operatorname{Aut}\left(\mathfrak{e}_{6}\right)$-structure which admits a reduction of structure group to a principal $E_{6^{-}}$ bundle, which defines $E$. This $\operatorname{Aut}\left(\mathfrak{e}_{6}\right)$-structure is well defined by an $\operatorname{Inn}\left(\mathfrak{e}_{6}\right)$ structure, except for the action of the outer involution $\sigma$.

Since $\operatorname{Inn}\left(\mathfrak{e}_{6}\right) \cong E_{6} / Z\left(E_{6}\right)$ and $Z\left(E_{6}\right) \cong \mathbb{Z}_{3}$, we have an exact sequence of groups

$$
1 \rightarrow \mathbb{Z}_{3} \rightarrow E_{6} \rightarrow \operatorname{Inn}\left(\mathfrak{e}_{6}\right) \rightarrow 1,
$$

from which we obtain that there exists $L \in H^{0}\left(X, \mathbb{Z}_{3}\right)$ such that $E^{\prime} \cong E \otimes L$ or $E^{\prime} \cong \sigma(E) \otimes L$, as we wanted to see.

The following is the main result of the section. We prove it here in detail because, although the proof is similar to the proof of Theorem 3 it introduces an important new step which makes use of Proposition 7.

Theorem 4. Let $M\left(E_{6}\right)$ be the moduli space of polystable principal $E_{6}$-bundles over the complex algebraic curve $X$. Let $\Phi: M\left(E_{6}\right) \rightarrow M\left(E_{6}\right)$ be an automorphism of $M\left(E_{6}\right)$. Then $\Phi$ is induced by an automorphism of the base curve $X$, an outer automorphism of $E_{6}$ and an element of $H^{1}\left(X, \mathbb{Z}_{3}\right)$.

Proof. We assume that the automorphism of $X$ induced by $\Phi$, given by Theorem 2 . is the identity.

Let $E$ be a stable and simple principal $E_{6}$-bundle, $\mathcal{H}: H^{0}\left(E\left(\mathfrak{e}_{6}\right) \otimes K\right) \rightarrow B$ be the Hitchin map with base $B$. For $r=2,5,6,8,9,12$, let $B_{r}$ be the summand $B_{r}=H^{0}\left(K^{r}\right)$ in the decomposition of $B$ (it is well defined by Lemma 2), and let 
$\mathcal{H}_{r}$ be the composition of the Hitchin map $\mathcal{H}$ with the projection map of $B$ over $B_{r}$. Let $f: B \rightarrow B$ be the automorphism of $B$ induced by $\Phi$. Let $x \in X$. The arguments which allow to prove Lemma 2 show that $\mathcal{H}_{r}\left(H^{0}\left(E\left(\mathfrak{e}_{6}\right) \otimes K(-x)\right)\right)$ is preserved by $f$, so $d \Phi$ preserves $\bigcap_{r=2,5,6,8,9,12} \mathcal{H}_{r}^{-1}\left(\mathcal{H}_{r}\left(H^{0}\left(E\left(\mathfrak{e}_{6}\right) \otimes K(-x)\right)\right)\right)$. From the form of the invariant polynomials, it is clear that the image of this set under the surjective map $\left.H^{0}\left(E\left(\mathfrak{e}_{6}\right) \otimes K\right) \rightarrow E\left(\mathfrak{e}_{6}\right) \otimes K\right|_{x}$ coincides with the nilpotent cone

$$
N(E)_{x}=\left\{\left.A \in E\left(\mathfrak{e}_{6}\right) \otimes K\right|_{x}: A \text { is nilpotent }\right\} .
$$

This is the fiber over $x$ of the nilpotent cone, $N(E)$, of $E$. We have constructed an isomorphism of the nilpotent bundles $N(E) \rightarrow N(\Phi(E)$ ) as bundles over $X$ (since the automorphism of the curve $X$ induced by $\Phi$ is the identity, by hypothesis). In $[9]$ it is proved that this automorphism induces an automorphism of the corresponding Borel flag varieties $\operatorname{Flag}(E)$ and $\operatorname{Flag}(\Phi(E))$. This automorphism induces a Lie algebra bundle isomorphism $E\left(\mathfrak{e}_{6}\right) \cong \Phi(E)\left(\mathfrak{e}_{6}\right)$. Now, from Proposition 7, there exists $L \in H^{1}\left(X, \mathbb{Z}_{3}\right)$ such that $\Phi(E) \cong E \otimes L$ or $\Phi(E) \cong \sigma(E) \otimes L$, where $\sigma$ is the outer involution of $E_{6}$. Since we have done all the construction assuming that the automorphism of $X$ induced by $\Phi$ is the identity, the result is proved when restricted to the open subset $M_{*}\left(E_{6}\right)$ of stable and simple principal $E_{6}$ bundles. Since this open subset is dense, the result holds.

Observe that the group $H^{1}\left(X, Z\left(E_{6}\right)\right) \cong H^{1}\left(X, \mathbb{Z}_{3}\right)$ is isomorphic to the third torsion of the Picard group of $X, \operatorname{Pic}(X)[3]$. We now formulate the main result of the section using this observation.

Theorem 5. Let $X$ be a complex algebraic curve of genus $g \geq 3$ and let $M\left(E_{6}\right)$ be the moduli space of polystable principal $E_{6}$-bundles over $X$. Then the group of automorphisms of $M\left(E_{6}\right)$ is isomorphic to

$$
\operatorname{Pic}(X)[3] \rtimes\left(\mathbb{Z}_{2} \times \operatorname{Aut}(X)\right) .
$$

The nontrivial element of $\mathbb{Z}_{2}$ acts on $\operatorname{Pic}(X)[3]$ by taking the dual of the bundle and $\operatorname{Aut}(X)$ acts on $\operatorname{Pic}(X)[3]$ by taking the pull-back.

Proof. In the proof of Theorem 4 we have established a surjective homomorphism of groups

$$
\operatorname{Aut}\left(M\left(E_{6}\right)\right) \rightarrow \operatorname{Out}\left(E_{6}\right) \times \operatorname{Aut}(X) \rightarrow 1
$$

which assigns to each isomorphism $\Phi$ of $M\left(E_{6}\right)$ the induced isomorphism of the curve $X$ given by Theorem 2 and the outer automorphism which arises from Theorem 4 Also from the proof of Theorem 4 it follows that the kernel of this homomorphism of groups is $H^{1}\left(X, Z\left(E_{6}\right)\right) \cong H^{1}\left(X, \mathbb{Z}_{3}\right)$. By Lemma 6 , the action of $H^{1}\left(X, \mathbb{Z}_{3}\right)$ on $M\left(E_{6}\right)$ is effective, so we have an exact sequence of groups

$$
1 \rightarrow H^{1}\left(X, \mathbb{Z}_{3}\right) \rightarrow \operatorname{Aut}\left(M\left(E_{6}\right)\right) \rightarrow \operatorname{Out}\left(E_{6}\right) \times \operatorname{Aut}(X) \rightarrow 1 .
$$

The groups $\operatorname{Out}\left(E_{6}\right) \cong \mathbb{Z}_{2}$ and $\operatorname{Aut}(X)$ clearly act on $H^{1}\left(X, \mathbb{Z}_{3}\right)$ as established in the statement of the theorem. Finally, observe that $\operatorname{Pic}(X)[3]$ is (after fixing a third root of unity) isomorphic to $H^{1}\left(X, \mathbb{Z}_{3}\right)$, so the result is proved. 


\section{SOME CONSEQUENCES}

Let $X$ be a complex projective irreducible curve of genus $g \geq 3$. Let $M\left(E_{6}\right)$ and $M\left(F_{4}\right)$ be the moduli spaces of polystable principal $E_{6}$-bundles and $F_{4}$-bundles over $X$, respectively. In a forthcoming paper 4 it is proved that the natural map between moduli spaces

$$
M\left(F_{4}\right) \rightarrow M\left(E_{6}\right)
$$

induced by the inclusion of groups $F_{4} \hookrightarrow E_{6}$ is injective. Therefore, there is at least one copy of $M\left(F_{4}\right)$ included in $M\left(E_{6}\right)$. Let $M_{0}$ be this copy. The following result is easily seen from Theorems 3 and 4

Proposition 8. Let $M\left(E_{6}\right)$ be the moduli space of principal $E_{6}$-bundles over $X$ and let $M_{0}$ be the subvariety of $M\left(E_{6}\right)$ defined in (13). Then any automorphism of $M_{0}$ is the restriction to $M_{0}$ of an automorphism of $M\left(E_{6}\right)$.

Proof. Let $\phi: M_{0} \rightarrow M_{0}$ be an automorphism of $M_{0}$. Since $M_{0} \cong M\left(F_{4}\right)$, from Theorem 3 there exists an automorphism $\alpha: X \rightarrow X$ such that $\phi=\alpha^{*}$. This automorphism $\alpha^{*}$ also induces an automorphism of $M\left(E_{6}\right)$, which we also call $\alpha^{*}$, such that the diagram

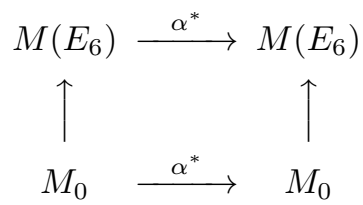

commutes. This proves the result.

Remark. The subset $M_{0} \cong M\left(F_{4}\right)$ is described in [3, Proposition 7.2] as the subvariety of fixed points of the action in $M\left(E_{6}\right)$ of the unique outer involution $\sigma$ of $E_{6}$. We then have that the map 13 gives a commutative diagram,

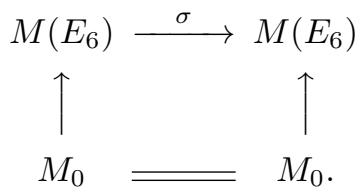

This shows that the induced automorphism of $M\left(E_{6}\right)$ given by Proposition 8 is not unique. Moreover, since every principal $E_{6}$-bundle fixed by $\sigma$ is strictly polystable ([3, Proposition 7.2]), $M_{0}$ lies in the strictly polystable locus of $M\left(E_{6}\right)$.

Proposition 8 and the discussion below show that any automorphism of $M_{0}$ is induced by an automorphism of $M\left(E_{6}\right)$, which is not unique, by restriction. We are now interested in studying the effect on $M_{0}$ of any automorphism of $M\left(E_{6}\right)$.

Proposition 9. Let $M\left(E_{6}\right)$ and $M\left(F_{4}\right)$ be the moduli spaces of principal $E_{6}$ bundles and $F_{4}$-bundles over $X$, respectively. Let $M_{0}$ be the subvariety of $M\left(E_{6}\right)$ defined in (13), which is isomorphic to $M\left(F_{4}\right)$. Let $L$ be a nontrivial element of $\operatorname{Pic}(X)[3]$. Let $f_{L}: M\left(E_{6}\right) \rightarrow M\left(E_{6}\right)$ be the automorphism of $M\left(E_{6}\right)$ induced by $L$ defined in 12 . Then there exist two other subvarieties of $M\left(E_{6}\right), M_{1}$ and 
$M_{2}$, with $M_{0} \cong M_{1} \cong M_{2} \cong M\left(F_{4}\right)$, such that the isomorphism $f_{L}$ restricts to isomorphisms of the form

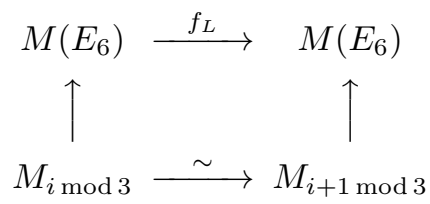

for $i=0,1,2$. Moreover, $M_{0}, M_{1}$ and $M_{2}$ fall in the strictly polystable locus of $M\left(E_{6}\right)$ and they are pairwise disjoint.

Proof. The subvarieties defined by $M_{1}=f_{L}\left(M_{0}\right)$ and $M_{2}=f_{L}^{2}\left(M_{0}\right)=f_{L^{2}}\left(M_{0}\right)$ satisfy the first part of the statement.

Now, if $E \in M_{0}$, then $E$ admits a copy of the trivial line bundle $\mathcal{O}$ as a subbundle and the exact sequence

$$
0 \rightarrow \mathcal{O} \rightarrow E \rightarrow E / \mathcal{O} \rightarrow 0
$$

splits. Then, the exact sequence

$$
0 \rightarrow L \rightarrow E \otimes L \rightarrow E / L \rightarrow 0
$$

also splits, so $E \otimes L=f_{L}(E)$ is strictly polystable. Since any element of $M_{1}$ is of this form, $M_{1}$ lies in the strictly polystable locus of $M\left(E_{6}\right)$. The same for $M_{2}$.

Finally, we will see that $M_{0}$ and $M_{1}$ are disjoint. Suppose, on the contrary, that they are not disjoint. Then there exists $E \in M_{0}$ such that $f_{L}(E) \in M_{0}$. There exists $E^{\prime} \in M\left(F_{4}\right)$ such that $E \cong E^{\prime} \oplus \mathcal{O}$. Since $f_{L}(E) \in M_{0}$, there exists $P_{1} \in M\left(F_{4}\right)$ such that

$$
\left(E^{\prime} \otimes L\right) \oplus L \cong P_{1} \oplus \mathcal{O} .
$$

It follows, since $L \neq \mathcal{O}$ and $P_{1}$ and $E^{\prime}$ are principal $F_{4}$-bundles, that $E^{\prime}$ and $P_{1}$ decompose as direct sum of vector bundles of the form

$$
E^{\prime} \otimes L \cong \mathcal{O} \oplus \mathcal{O} \oplus E^{\prime \prime}
$$

and

$$
P_{1} \cong L \oplus L^{-1} \oplus P_{2}
$$

Of course, for these decompositions, we consider the underlying vector bundles of $E^{\prime}$ and $P_{1}$. We then have, from (14), that

$$
E^{\prime \prime} \oplus \mathcal{O} \oplus \mathcal{O} \oplus L \cong P_{2} \oplus L \oplus L^{-1} \oplus \mathcal{O},
$$

that is,

$$
E^{\prime \prime} \oplus \mathcal{O} \cong P_{2} \oplus L^{-1} .
$$

If we iterate enough the process, we obtain that there exist $r, s$ with $2 r+2 s=26$ (so $r+s=13$ ) such that

$$
E^{\prime} \cong\left(\oplus^{r}\left(L^{2} \oplus\left(L^{2}\right)^{-1}\right)\right) \oplus\left(\oplus^{s}(\mathcal{O} \oplus \mathcal{O})\right)
$$

and there exist $r^{\prime}, s^{\prime}$ with $r^{\prime}+s^{\prime}=13$ such that

$$
P_{1} \cong\left(\oplus^{r^{\prime}}\left(L \oplus L^{-1}\right)\right) \oplus\left(\oplus^{s^{\prime}}(\mathcal{O} \oplus \mathcal{O})\right) .
$$


It is easy to see, from (14, that

$$
\begin{aligned}
r^{\prime} & =s+1, \\
r^{\prime} & =s .
\end{aligned}
$$

This is a contradiction, so the last part of the result is proved.

Acknowledgments. The author would like to thank the referee for very useful comments that helped to improve the paper.

\section{REFERENCES}

[1] J.F. Adams, Lectures on exceptional Lie groups, University of Chicago Press, 1996. MR 1428422

[2] M. Adler and P. van Moerbeke, Completely integrable systems, Euclidean Lie algebras and curves, Adv. in Math. 38 (3) (1980), 267-317. MR 0597729

[3] A. Antón, Automorphisms of the moduli space of principal G-bundles induced by outer automorphisms of $G$, Math. Scand. (In press).

[4] A. Antón, The moduli space of $E_{6}$-Higgs bundles over an algebraic curve, In preparation.

[5] I. Biswas, T.L. Gómez and V. Muñoz, Automorphisms of moduli spaces of symplectic bundles, Internat. J. Math. 23 (2012), no. 5, 1250052, 27 pp. MR 2914655

[6] I. Biswas, T.L. Gómez and V. Muñoz, Automorphisms of moduli spaces of vector bundles over a curve, Expo. Math. 31 (2013), no. 1, 73-86. MR 3035121.

[7] C. Chevalley, Invariants of finite groups generated by reflections. Amer. J. Math. 77 (4) (1955), 778-782. MR 0072877

[8] R. Donagi, Decomposition of spectral covers. Astérisque 218 (1993), 145-175. MR 1265312.

[9] J. Draisma, H. Kraft and J. Kuttler, Nilpotent subspaces of maximal dimension in semisimple Lie algebras, Compos. Math. 142 (2006), 464-476. MR 2218906

[10] G. Faltings, Stable G-bundles and projective connections, J. Algebraic Geom. 2 (1993), 507568. MR 1211997.

[11] J.C. Ferrar, Lie algebras of type $E_{6}$, J. Algebra 13 (1969), 57-72. MR 0263881.

[12] J.C. Ferrar, Lie algebras of type $E_{6}$, II, J. Algebra 52 (1978), 201-209. MR 0480651

[13] N. Hitchin, Stable bundles and integrable systems. Duke Math. J. 54 (1) (1987), 91-114. MR 0885778

[14] J.-M. Hwang and S. Ramanan, Hecke curves and Hitchin discriminant, Ann. Sci. École Norm. Sup. (4) 37 (2004), no. 5, 801-817. MR 2103475

[15] A. Kouvidakis and T. Pantev, The automorphism group of the moduli space of semistable vector bundles, Math. Ann. 302 (1995), 225-268. MR 1336336

[16] D. Mumford and P. Newstead, Periods of a moduli space of bundles on curves, Amer. J. Math. 90 (1968), 1200-1208. MR 0234958

[17] A.L. Onishchik and E.B. Vinberg (Eds.), Lie Groups and Lie Algebras III, Encyclopedia of Mathematical Sciences, Volume 41, Springer-Verlag, 1994. MR 1349140.

[18] A. Ramanathan, Stable principal bundles on a compact Riemann surface, Math. Ann. 213 (1975), 129-152. MR 0369747

[19] A. Ramanathan, Moduli for principal bundles over algebraic curves. I, Proc. Indian Acad. Sci. Math. Sci. 106 (1996), no. 3, 301-328. MR 1420170.

[20] A. Ramanathan, Moduli for principal bundles over algebraic curves. II, Proc. Indian Acad. Sci. Math. Sci. 106 (1996), no. 4, 421-449. MR 1425616. 
[21] R. Rubio, Exceptional G-Higgs bundles, DEA Thesis, Universidad Autónoma de Madrid, 2007.

[22] O. Serman, Moduli spaces of orthogonal and symplectic bundles over an algebraic curve, Compos. Math. 144 (2008), 721-733. MR 2422347

[23] T.A. Springer and F.D. Veldkamp, Octonions, Jordan algebras and exceptional groups, Springer Monographs in Mathematics, Springer-Verlag, 2000. MR 1763974.

[24] I. Yokota, Realizations of involutive automorphisms $\sigma$ and $G^{\sigma}$ of exceptional linear groups $G$, part I, $G=G_{2}, F_{4}$ and $E_{6}$, Tsukuba J. Math. 14 (1) (1990), 185-223. MR 1063845

\author{
Álvaro Antón Sancho \\ Department of Mathematics and Experimental Sciences, School of Education 'Fray Luis de \\ León’ (Catholic University of Ávila), c/ Tirso de Molina, 44, 47010 Valladolid, Spain \\ alvaro.anton@frayluis.com
}

Received: January 23, 2017

Accepted: May 16, 2017 